\title{
Rendering Bio-Inert Low Density Polyethylene Amenable For Biodegradation Via Fast High Throughput Reactive Extrusion Assisted Oxidation
}

\section{Pablo Ferrero}

AIMPLAS

Olivia A. Attallah ( $D$ oadly@ait.ie )

Athlone Institute of Technology https://orcid.org/0000-0002-6449-5108

\section{Miguel Ángel Valera}

AIMPLAS

Ivana Aleksic

University of Belgrade: Univerzitet u Beogradu

Muhammad Azeem

Athlone Institute of Technology

Jasmina Nikodinovic-Runic

University of Belgrade: Univerzitet u Beogradu

Margaret Brennan Fournet

Athlone Institute of Technology

\section{Research}

Keywords: low density polyethylene, reactive extrusion, high throughput, bio-adhesion, Box Behnken design

Posted Date: November 1st, 2021

DOI: https://doi.org/10.21203/rs.3.rs-1016043/v1

License: (a) (1) This work is licensed under a Creative Commons Attribution 4.0 International License. Read Full License

Version of Record: A version of this preprint was published at Journal of Polymers and the Environment on February 11th, 2022. See the published version at https://doi.org/10.1007/s10924-022-02400-w. 


\section{Abstract}

An energy efficient high throughput pre-treatment of low density polyethylene (LDPE) using a fast reactive extrusion (REX) assisted oxidation technique followed by bacterial attachment as an indicator for bioamenability was studied. Silicon dioxide $\left(\mathrm{SiO}_{2}\right)$ was selected as a model oxidizing and catalytic reagent with the REX process demonstrated to be effective both in the presence and absence of the catalyst. Optimized 5-minute duration pretreatment conditions were determined using Box-Behnken design (BBD) with respect to screws speed, operating temperature, and concentration of $\mathrm{SiO}_{2}$. The crystallinity index, carbonyl index and weight loss (\%) of LDPE were used as the studied responses for BDD. FTIR and DSC spectra of the residual LDPE obtained after pretreatment with the REX assisted oxidation technique showed a significant increase in residual LDPE carbonyl index from 0 to 1.04 and a decrease of LDPE crystallinity index from $29 \%$ to $18 \%$. Up to 5 -fold molecular weight reductions were also demonstrated using GPC. Optimum LDPE pretreatment with a duration of 5 minutes was obtained at low screw speed (50 rpm), operating temperature of $380-390 \otimes \mathrm{C}$ and variable concentration of $\mathrm{SiO}_{2}(0$ and $2 \%(\mathrm{w} / \mathrm{w}))$ indicating that effective pretreatment can occur under noncatalytic and catalysed conditions. Biofilms were successfully formed on pretreated LDPE samples after 14 days of incubation.

Furthermore, the technique proposed in this study is expected to provide a high throughput approach for pretreatment of pervasive recalcitrant PE based plastics to reduce their bio inertness.

\section{Introduction}

Plastic production has been in continuous growth since their discovery. To date, the global plastic production has reached 368 million tons giving direct employment to more than 1.56 million people in Europe [1]. Their remarkable properties and high performances at low market prices mean that plastic materials are now common-place across the globe. The pervading use of plastics and the absence of suitable treatments at the end of their life cycle, continues to lead to damaging pollution of the natural environment. An important example of such consumption is plastic bags which is estimated to be in the range of 500 billion to 1 trillion plastic bags each year worldwide [2]. Most of these plastic bags are made from low density polyethylene (LDPE); a polymer widely used in food packaging and agriculture applications. One of the main characteristics of LDPE is its inertness, which despite being important for maintaining the stability of the packaged material, critically hinders the natural biodegradation of LDPE leading to long-term persistence within the environmental. LDPE hence constitutes a major environmental pollutant with reported threats to wildlife such as blockages in the intestines of different marine animals and birds [3,4].

The biodegradation of polymers relies mainly on the hydrolytic extracellular enzymes produced by microorganisms to create lower molecular weight intermediates that can be assimilated and metabolized by the microorganisms [5]. Chemical and physical modification of the plastic surface including photooxidation, and ultrasonication enhance their biodegradation via surface modification [5]. As the initial step microorganisms attach to the surfaces and usually form biofilms, which are complex 
microorganism life structures and communities. It has been shown recently that environmental bacteria consortia formed biofilms on polyethylene terephthalate (PET) polyester plastic, inducing molecular changes on the plastic surface and indicating the initial stages of plastic degradation [6]. LDPE is one of the most bio-inert of the petroleum based plastics and considerable research has been focused on developing methods to facilitate LDPE biodegradation. It is recognized that the high hydrophobicity, high molecular weight and absence of chemical groups [7] are the biggest challenges preventing polyethylene biodegradation. The combination of mechan-thermal and green chemical pretreatments to modify the physicochemical properties of LDPE and enable amenability to microorganism and enzymatic interactions is a highly favorable route towards the achievement of biological recyclability of this polymer. A limited number of studies have shown that pretreatment which oxidizes the surface of LPDE can lead to compatibility with microorganisms for biodegradation $[8,9]$. Hypotheses of the facilitation of carbonyl group formation of microorganism attack during LDPE oxidation in order to degrade LDPE have been previously proposed $[10,11]$. Other studies have shown that using additives in polyethylene (PE) films can induce films' oxidation causing fragmentation, decreasing the molecular weight and increasing in wettability of the films thereby, leading to enhanced PE biodegradability [12].

At the industrial level, an important factor to take into account is that continuous processes and reaction systems are desired for operational reasons rather batch ones [13]. Therefore, pretreatment processes which can be adopted for continuous operation and pave the way for a more sustainable disposal of LDPE at the industrial scale are urgently required. Reactive extrusion (REX) is a technique that has been recently used for the surface modification of polymers and manipulation of their properties [14]. REX techniques can be operated in continuous modes to provide high throughput polymer treatment, hence making it industrially favorable. Several studies have evaluated the use of REX as a depolymerization technique for LDPE. However, most of the REX reactions were carried out under very harsh conditions to allow complete depolymerization of LDPE. For example, Wallis et al. [15] studied the thermal degradation described as random breakage of polyethylene in a reactive extruder at various screw speeds (4-16 $\mathrm{rpm}$ ) with reaction temperatures of $400^{\circ} \mathrm{C}$ and $425^{\circ} \mathrm{C}$ and polymer melting and residence times of 2 to 3 hours. In another study, Shah et al. [16] also tested the use of different catalyst for the depolymerization of LDPE in a reactive extruder at temperatures ranging from 250 to $400^{\circ} \mathrm{C}$ with a reaction time of 60 min. Concretely, they used catalysts as $\mathrm{SiO}_{2}, \mathrm{Al}_{2} \mathrm{O}_{3}, \mathrm{CaC}_{2}, \mathrm{ZnO}$ and $\mathrm{MgO}$ during the depolymerization reaction and found that $\mathrm{SiO}_{2}$ was the most efficient for the conversion process.

In this study, pre-treatment of LDPE using REX assisted oxidation technique followed by assessment of bacterial adherence and attachment as an indicator for biodegradation is employed. The fast processing on a timescale of 5 minutes is highly favourable in terms of the energy consumption. Silicon dioxide $\left(\mathrm{SiO}_{2}\right)$ was selected as a model oxidizing and catalytic reagent in the REX process which facilitated LDPE pretreatment in a high throughput fashion. Optimized pretreatment conditions were determined using Box-Behnken design (BBD) with respect to screw speed, operating temperature, and concentration of $\mathrm{SiO}_{2}$. The crystallinity index, carbonyl index and weight loss (\%) of LDPE were used as the studied responses for BDD. The responses were chosen based on the desirable characteristics for materials 
undergoing biodegradation as low crystallinity, high hydrophilicity due to carbonyl groups and low mass material are important indications for enhanced biodegradation. Oxidized residue of LDPE resulting from the optimized pretreatment process were further exposed to bacterial isolates to assess the efficiency of the proposed pretreatment in enhancing bacterial attachment to LDPE. The success of the technique proposed in this study is expected to provide a sustainable high throughput approach for PE based plastics to improve their biodegradation.

\section{Experimental \\ 2.1 Material}

LDPE was purchased from Lupolen $1800 \mathrm{~S}$ grade (Lyondel Basell, Netherlands). Silicon dioxide $\left(\mathrm{SiO}_{2}\right)$ (Ibersil@ A-400) obtained from IQE. All other chemicals were of analytical grade. Crystal violet (hexamethylpararosaniline chloride) and other microbiological media components were obtained from Sigma-Aldrich (Munich, Germany).

\subsection{Pretreatment of LDPE in a reactive extruder}

The pretreatment experiments were performed by extruding $5.0 \mathrm{gm}$ of powdered LDPE with varied amounts of $\mathrm{SiO}_{2}$ in MiniLab II HAAKE Rheomex CTW5 co-rotating conical twin-screw extruder at specified screw speed and reaction temperature. The retention time of the extrusion process was fixed at 5 min then samples were taken for further analysis. The weight loss of LDPE was calculated using following equation:

LDPE Weight loss $(\%)=($ W1 -W2) W1 $\times 100(1)$

where $W_{1}$ was LDPE initial weight and $W_{2}$ was the weight of LDPE residual after REX pretreatment.

\subsection{Design of experiment}

Two separate three-factor, three-level Box-Behnken designs (Design Expert, trial version 10.0.5.0, StatEase Inc., Minneapolis, MN) were implemented to optimize LDPE pretreatment using REX technique. The design involved 15 experimental runs with three center points. The central points were repeated three times to confirm the normal distribution of the results. The independent factors were screws speed $\left(\mathrm{X}_{1}\right)$, operating temperature $\left(X_{2}\right)$, and Concentration of $\mathrm{SiO}_{2}\left(X_{3}\right)$ and were varied at three levels. The responses were taken as the calculated crystallinity index, carbonyl index and weight loss (\%) of LDPE. The selected ranges for each independent factor were based on available literature and preliminary experiments (Table 1). 
Table 1

Variables and levels in Box-Behnken experimental design for model LDPE pretreatment

\begin{tabular}{|llll|}
\hline & level \\
\hline Independent variables & -1 & 0 & 1 \\
\hline $\mathrm{X}_{1}$ : Screws speed (RPM) & 50 & 100 & 150 \\
\hline $\mathrm{X}_{2}:$ Temperature (खC) & 300 & 350 & 400 \\
\hline $\mathrm{X}_{3}$ : Concentration of $\mathrm{SiO}_{2}(\mathrm{wt} \%(\mathrm{w} / \mathrm{w}))$ & 0 & 1 & 2 \\
\hline
\end{tabular}

\subsection{Assessment of bacterial attachment and biofilm formation}

Pseudomonas aeruginosa ATCC 10332 and defined consortium of three different bacterial strains $(P$. aeruginosa ATCC 10332 and two Bacillus sp., namely KIA-19 and FIA-30, form the laboratory collection) were used to assess their ability to adhere and form biofilms on LDPE samples (Optimized 1, Optimized 2, untreated control and disposable $1 \mathrm{~mL}$ automatic pipette tip widely used in the research laboratories). Plastic was cut into pieces of approximately the same size and weight $(2.5 \mathrm{~cm} \times 1 \mathrm{~cm} ; 200 \mathrm{mg})$. Plastic samples were briefly rinsed with ethanol $(70 \%, \mathrm{v} / \mathrm{v})$, dried and aseptically transferred to sterile plastic tubes (10 mL volume). Standard nutrient rich Luria Bertani (LB) medium (1\% (w/v) tryptone, $0.5 \%(\mathrm{w} / \mathrm{v})$ yeast extract and $1 \%(\mathrm{w} / \mathrm{v}) \mathrm{NaCl})$ was added to the tubes $(2 \mathrm{~mL})$ and this was inoculated with the overnight culture of bacteria $(0.1 \%, \mathrm{v} / \mathrm{v})$. Tubes were incubated at $37^{\circ} \mathrm{C}$, on rotary shaker $70-100 \mathrm{rpm}$, for 14 days.

After incubation, the amount of formed biofilms was quantified by crystal violet staining (CV) as previously described in [17] with some minor modifications. Plastic pieces were rinsed with distilled water, air dried and dyed with $0.1 \%(\mathrm{w} / \mathrm{v}) \mathrm{CV}$ solution for $20 \mathrm{~min}$, rinsed with distilled water and air dried. For distaining $5 \mathrm{~mL}$ of $30 \%(\mathrm{v} / \mathrm{v})$ acetic acid solution was placed into tubes and plastic pieces were added, vigorously mixed and left at $4^{\circ} \mathrm{C}$ overnight. Absorbance of the solution was measured at $590 \mathrm{~nm}$ using UV-vis spectrophotometer Ultrospec 3300pro (Amersham Biosciences, USA) as an indicator of the amount of biofilms formed on the plastic pieces. Plastic pieces were rinsed with water, dried and their mass and carbonyl and crystallinity indices were determined.

\subsection{Instrumental Characterization}

The LDPE samples before and after REX pretreatment were analysed by FTIR spectroscopy (Perkin Elmer, UK) at a spectral region of $4000-600 \mathrm{~cm}^{-1}$. Carbonyl Index was determined based on the obtained results using baseline method. Ratios of carbonyl peak intensity at $1716 \mathrm{~cm}^{-1}$ to that of the normal $\mathrm{C}-\mathrm{H}$ bonding mode at $1376 \mathrm{~cm}^{-1}$ in LDPE were calculated as follows [18], 
Carbonyl index $=\frac{\text { Absorption at } 1716 \mathrm{~cm}^{-1}}{\text { Absorption at } 1376 \mathrm{~cm}^{-1}}$

The thermal behaviour of the LDPE samples was evaluated by a DSC Perkin Elmer 4000 (Perkin Elmer Washington, Ma, USA) with Pyris Software (Version 13.3.1) under an inert nitrogen stream. About $10 \mathrm{mg}$ of specimen was sealed in an aluminum pan and the DSC scans were recorded while heating from 30 $150{ }^{\circ} \mathrm{C}$ at a heating rate of $10^{\circ} \mathrm{C} / \mathrm{min}$, and then cooled to $30^{\circ} \mathrm{C}$. The crystallinity index was calculated according to the following Equation [19],

Crystallinity index $=\left(\Delta H_{m} / W \Delta H_{m o}\right) \times 100(3)$

where $\Delta \mathrm{H}_{\mathrm{m}}(\mathrm{J} / \mathrm{g})$ is the heat of fusion of the LDPE sample. $\Delta \mathrm{H}_{\mathrm{m} 0}$ is the heat of fusion for completely crystalline LDPE $(293 \mathrm{~J} / \mathrm{g})[20]$ and $\mathrm{W}(\mathrm{g})$ is the weight fraction of residual LDPE in the samples.

\section{Results And Discussion}

\subsection{Experimental design}

The model of LDPE pretreatment using REX was studied by response surface methodology. In the current study, the experimental runs were carried out based on the design plan proposed for the studied parameters (screws speed, operating temperature and concentration of $\mathrm{SiO}_{2}$ ). After each run, the crystallinity index, carbonyl index and weight loss (\%) of LDPE were calculated and presented as responses for each run as shown in Table 2. 
Table 2

Experimental matrix and observed responses for LDPE pretreatment in BBD

\begin{tabular}{|c|c|c|c|c|c|c|}
\hline \multicolumn{4}{|c|}{ Independent variable } & \multicolumn{3}{|c|}{ Dependent variable } \\
\hline Run & $\mathrm{X}_{1}(\mathrm{RPM})$ & $\mathrm{X}_{2}(\mathbb{}(\mathrm{C})$ & $X_{3}(w t \%)$ & $\mathrm{Y}_{1}$ & $Y_{2}$ & $\begin{array}{l}Y_{3} \\
(\%)\end{array}$ \\
\hline 1 & 100 & 350 & 1 & 22.34 & 0.85 & 64.03 \\
\hline 2 & 100 & 350 & 1 & 22.70 & 0.92 & 64.16 \\
\hline 3 & 50 & 400 & 1 & 15.97 & 0.06 & 56.79 \\
\hline 4 & 150 & 300 & 1 & 24.16 & 0.76 & 61.90 \\
\hline 5 & 100 & 300 & 0 & 23.58 & 1.94 & 63.16 \\
\hline 6 & 50 & 350 & 2 & 22.32 & 1.38 & 63.84 \\
\hline 7 & 150 & 350 & 2 & 25.12 & 2.03 & 55.29 \\
\hline 8 & 100 & 300 & 2 & 25.06 & 0.41 & 60.98 \\
\hline 9 & 150 & 400 & 1 & 20.35 & 0.57 & 50.33 \\
\hline 10 & 150 & 350 & 0 & 22.67 & 2.03 & 64.35 \\
\hline 11 & 100 & 350 & 1 & 23.00 & 0.80 & 64.35 \\
\hline 12 & 50 & 350 & 0 & 23.40 & 2.16 & 62.62 \\
\hline 13 & 100 & 400 & 2 & 17.51 & 1.00 & 50.10 \\
\hline 14 & 100 & 400 & 0 & 16.82 & 0.48 & 56.48 \\
\hline 15 & 50 & 300 & 1 & 26.00 & 0.77 & 62.53 \\
\hline
\end{tabular}

The studied responses were then tested against different regression models to determine the best-fitting mathematical model and the significance of varying the process parameters. The quadratic model was chosen as the best fitting model for the studied responses in comparison to the other models. The relationship between the crystallinity index $\left(\mathrm{Y}_{1}\right)$ and carbonyl index $\left(\mathrm{Y}_{2}\right)$ and the studied parameters; screws speed $\left(X_{1}\right)$, operating temperature $\left(X_{2}\right)$, and Concentration of $\mathrm{SiO}_{2}\left(X_{3}\right)$ is demonstrated in Table 3 .

For crystallinity index $\left(Y_{1}\right)$, the coefficients of the quadratic model equation indicated that the increase in operating temperature led to a significant decrease in the crystallinity index of LDPE residues while the increase in the screws speed and concentration of $\mathrm{SiO}_{2}$ showed a positive effect. The screws speed's interactions with both operating temperature and concentration of $\mathrm{SiO}_{2}$ also showed a significant 
positive efficacy on crystallinity index. On the other hand, the interaction between the operating temperature and concentration of $\mathrm{SiO}_{2}$ showed a negative effect on crystallinity index. Such results elaborate that all the studied factors had significant effects on the crystallinity index of treated LDPE residue. Despite the factorial levels' values, all the studied factors led to the creation of amorphous LDPE causing a decrease in the crystallinity index of treated LDPE samples which had an original crystallinity index of 29.20. Thus, making the LDPE residue favorable for microbial biodegradation.

For carbonyl index $\left(\mathrm{Y}_{2}\right)$, both the operating temperature and concentration of $\mathrm{SiO}_{2}$ showed a significant negative effect on the carbonyl index values while the increase in screws speed had a positive effect. Alternatively, the screws speed interaction with both the operating temperature and concentration of $\mathrm{SiO}_{2}$ showed significant positive effects on the carbonyl index. Thus, based on the obtained results, it can be indicated that the interactions of the studied factors led to an increase in LDPE oxidative degradation which was observed through the increase in the carbonyl index of the treated LDPE residues.

Percentage of LDPE weight loss $\left(\mathrm{Y}_{3}\right)$ was used as a response to assess LDPE initial degradation after REX. In Table 3, it can be observed that all the independent variables and their interactions influenced LDPE weight loss significantly with a P-value exceeding 0.05 . Additionally, the coefficients of the model equation showed that the increase in all the studied independent variables and their interactions above a certain level led to a decrease in LDPE weight loss. Such finding indicate that the weight loss of LDPE is not necessarily increased with high levels of screws speed, operating temperature or concentration of $\mathrm{SiO}_{2}$; yet, it requires careful adjustment of these factors' levels in order to obtain the desired initial degradation percentage.

Table 3: Statistical analysis of measured responses for LDPE pretreatment 


\begin{tabular}{|c|c|c|c|c|}
\hline Fitting model & Factors & Coefficient & P-value & ANOVA \\
\hline \multirow[t]{10}{*}{ Crystallinity index $\left(\mathrm{Y}_{1}\right)$} & Intercept & 22.68 & & \multirow{10}{*}{$\begin{array}{l}F=207.43, \\
R^{2}=0.9925, \text { Model } P \text {-value } \\
\bigotimes 0.0001, \\
P \text {-value of lack of fit }=0.41\end{array}$} \\
\hline & $x_{1}$ & 0.58 & 0.0017 & \\
\hline & $x_{2}$ & -3.52 & $\begin{array}{l}<.0001 \\
0.000\end{array}$ & \\
\hline & $x_{3}$ & 0.44 & 0.0053 & \\
\hline & $x_{1} x_{2}$ & 1.56 & $\begin{array}{l}< \\
0.0001\end{array}$ & \\
\hline & $x_{1} x_{3}$ & 0.88 & 0.0012 & \\
\hline & $x_{2} x_{3}$ & -0.2 & 0.1976 & \\
\hline & $x_{1}^{2}$ & 0.79 & 0.0023 & \\
\hline & $x_{2}^{2}$ & -1.85 & $\begin{array}{l}< \\
0.0001\end{array}$ & \\
\hline & $x_{3}{ }^{2}$ & -0.09 & 0.5442 & \\
\hline \multirow[t]{10}{*}{ Carbonyl index $\left(\mathrm{Y}_{2}\right)$} & Intercept & 0.86 & & \multirow{10}{*}{$\begin{array}{l}F=247.60, \\
R^{2}=0.9937, \text { Model } P \text {-value } \\
=\otimes 0.0001, \\
P \text {-value of lack of fit }=0.62\end{array}$} \\
\hline & $x_{1}$ & 0.13 & 0.0010 & \\
\hline & $x_{2}$ & -0.22 & $\begin{array}{l}< \\
0.0001\end{array}$ & \\
\hline & $x_{3}$ & -0.22 & $\begin{array}{l}<.0001 \\
0.00\end{array}$ & \\
\hline & $x_{1} x_{2}$ & 0.13 & 0.0045 & \\
\hline & $x_{1} x_{3}$ & 0.19 & 0.0007 & \\
\hline & $x_{2} x_{3}$ & 0.51 & $\begin{array}{l}< \\
0.0001\end{array}$ & \\
\hline & $x_{1}^{2}$ & 0.31 & $\begin{array}{l}< \\
0.0001\end{array}$ & \\
\hline & $x_{2}^{2}$ & -0.63 & $\begin{array}{l}<.0001 \\
0.00\end{array}$ & \\
\hline & $x_{3}^{2}$ & 0.73 & $\begin{array}{l}< \\
0.0001\end{array}$ & \\
\hline & Intercept & 64.18 & & $F=1461.89$ \\
\hline
\end{tabular}




\begin{tabular}{|c|c|c|c|c|}
\hline \multirow[t]{9}{*}{$\begin{array}{l}\text { LDPE weight loss (\%) } \\
\left(\mathrm{Y}_{3}\right)\end{array}$} & $x_{1}$ & -1.74 & $\begin{array}{l}<.0001 \\
0.00\end{array}$ & \multirow{9}{*}{$\begin{array}{l}R^{2}=0.9989, \text { Model } P \text {-value } \\
=\rrbracket 0.0001, \\
P \text {-value of lack of fit }=0.52\end{array}$} \\
\hline & $x_{2}$ & -4.36 & $\begin{array}{l}<.0001 \\
0.000\end{array}$ & \\
\hline & $x_{3}$ & -2.05 & $\begin{array}{l}<.0001 \\
0.00\end{array}$ & \\
\hline & $x_{1} x_{2}$ & -1.46 & $\begin{array}{l}< \\
0.0001\end{array}$ & \\
\hline & $\mathrm{x}_{1} \mathrm{x}_{3}$ & -2.57 & $\begin{array}{l}< \\
0.0001\end{array}$ & \\
\hline & $x_{2} x_{3}$ & -1.05 & $\begin{array}{l}< \\
0.0001\end{array}$ & \\
\hline & $x_{1}{ }^{2}$ & -1.22 & $\begin{array}{l}< \\
0.0001\end{array}$ & \\
\hline & $x_{2}^{2}$ & -5.07 & $\begin{array}{l}< \\
0.0001\end{array}$ & \\
\hline & $x_{3}^{2}$ & -1.43 & $\begin{array}{l}< \\
0.0001\end{array}$ & \\
\hline
\end{tabular}

$\mathrm{X}_{1}$ : Screws speed, $\mathrm{X}_{2}$ : Operating temperature, $\mathrm{X}_{3}$ : Concentration of $\mathrm{SiO}_{2}, \mathrm{Y}_{1}$ : Crystallinity index, $\mathrm{Y}_{2}$ : Carbonyl index, $Y_{3}$ : Weight loss (\%) of LDPE

The adequacy of the proposed model to describe the crystallinity index, carbonyl index and weight loss of treated LDPE residues was evaluated and the results are demonstrated in Table 3. A sequential test was performed and the obtained quadratic model F-values (207.43 for crystallinity index, 247.60 for carbonyl index and 14611.89 for LDPE weight loss) were large compared to other model terms values in the equation. Thus, the proposed experimental systems for all responses can be modeled effectively. Based on the statistics test, high coefficients of determination were observed for all studied responses. The adjusted $R^{2}$ values were calculated to be $0.9925,0.9937$ and 0.9989 for crystallinity index, carbonyl index and LDPE weight loss respectively).

Analysis of variance (ANOVA) was also applied to determine the significance of the model at a $95 \%$ confidence interval. A model is said to be significant if the probability value ( $p$-value) is $₫ 0.05$. The $p$ values demonstrated in Table $\mathbf{3}$ for crystallinity index, carbonyl index and LDPE weight loss indicated that these responses fitted the model well. From the lack-of-fit test the response showed a highly desirable non-significant lack-of-fit ( $\mathrm{p} \otimes 0.1)$ with $p$-values of 0.41 for crystallinity index, 0.62 for carbonyl index and 0.52 for LDPE weight loss.

\subsection{Response surface analysis}

Response surface graphical plots were generated between the responses obtained for LDPE pretreatment and the studied independent variables to estimate the effect of combinations of these variables on the 
studied responses. The 3-D and contour plots for crystallinity index, carbonyl index and LDPE weight loss (\%) are demonstrated in Figures 1, 2 and 3. Figure 1 illustrates the dependence of the studied responses on screws speed and operating temperature. It can be observed that high levels of operating temperature and low levels of screws speed resulted in a significant decrease in crystallinity index. On the other hand, increased carbonyl index and LDPE weight loss percentage were detected at 320 to $370 \mathbb{}$ C temperature range along all levels of screws speed. The values of both responses started to decrease at higher temperatures indicating the importance of adjusting the levels of temperature to obtain desirable degree of degradation. Thus, based on the obtained results, low screws speed and moderately elevated temperatures are required for LDPE oxidative degradation.

Figure 2 shows the dependence of the studied responses on screws speed and concentration of $\mathrm{SiO}_{2}$. $\mathrm{The}$ lowest crystallinity index was observed at low levels of both screws speed and concentration of $\mathrm{SiO}_{2}$ while increased values of carbonyl index were observed at low levels of $\mathrm{SiO}_{2}$ concentration and along all levels of screws speed. On the other hand, high concentrations of $\mathrm{SiO}_{2}$ led to elevated LDPE weight loss along all levels of screws speed where $\mathrm{SiO}_{2}$ acted as a catalyst in the LDPE degradation process. Therefore, it can be indicated that low levels of both $\mathrm{SiO}_{2}$ concentration and screws speed are able to produce desirable results for the crystallinity index and carbonyl index with respect to biodegradation. Nevertheless, high concentrations of $\mathrm{SiO}_{2}$ is also necessary to increase the rate of LDPE degradation during the REX process producing a residue of lower mass that can be more easily handled during biodegradation.

Figure 3 demonstrates the dependence of the studied responses on operating temperature and concentration of $\mathrm{SiO}_{2}$. It can be observed that the increase in operating temperature led to a decrease in crystallinity index of LDPE residues despite the concentration of $\mathrm{SiO}_{2}$ which indicates that the temperature has the upper hand in controlling the crystallinity of the treated LDPE samples. The same behavior was observed on LDPE weight loss where varying the $\mathrm{SiO}_{2}$ concentration didn't show a significant difference in the degradation of LDPE upon interaction with operating temperature. Moreover, the highest carbonyl index and LDPE weight loss (\%) were observed at a temperature range from 300 to

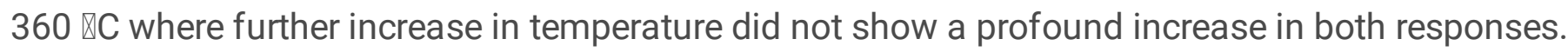

\subsection{Optimization of LDPE pretreatment via REX technique}

All three responses were optimized simultaneously using BBD optimization. Pretreatment optimum conditions were chosen with the aim of attaining maximum initial LDPE degradation and enhancement of residual LDPE biodegradation post pretreatment. Based on the BBD results, maximum LDPE weight loss was observed with LDPE residues of high crystallinity index and high carbonyl index. Alternatively, based on literature review, enhanced LDPE biodegradation can be achieved through high carbonyl index and low crystallinity index residues [21]. Bearing such necessity in mind, the pretreatment conditions were adjusted to attain minimum crystallinity index and maximum carbonyl index and weight loss of LDPE, as 
shown in Table 4. A total of 40 optimized solutions were obtained. The selected solutions were determined according to their success to attain an acceptable desirability greater than 0.5 for the studied responses. Two batch experiments were carried out for LDPE pretreatment using the optimized conditions and the three responses were evaluated to validate the predicted model factors and responses. The response values (predicted and observed) for the optimized conditions are recorded in Table 4. The model was proven to be validated since a fine agreement existed between the predicted and observed results. This indicates the success of the BBD for the evaluation and optimization of the proposed LDPE pretreatment process.

Table 4

The optimized LDPE pretreatment process with observed and predicted response values

\begin{tabular}{|c|c|c|c|c|c|}
\hline \multirow[t]{2}{*}{ Independent Variable } & & \multicolumn{4}{|c|}{ Optimized level } \\
\hline & & \multicolumn{2}{|c|}{ Optimized 1} & \multicolumn{2}{|c|}{ Optimized 2} \\
\hline $\mathrm{X}_{1}$ : Screws speed (RPM) & & \multicolumn{2}{|l|}{50} & \multicolumn{2}{|l|}{50} \\
\hline $\mathrm{X}_{2}$ : Operating temperature $(\mathbb{\nabla C})$ & & \multicolumn{2}{|l|}{380} & \multicolumn{2}{|l|}{390} \\
\hline $\begin{array}{l}\mathrm{X}_{3} \text { : Concentration of } \mathrm{SiO}_{2}(\mathrm{wt} \% \\
(\mathrm{w} / \mathrm{w}))\end{array}$ & & \multicolumn{2}{|l|}{0} & \multicolumn{2}{|l|}{2} \\
\hline Overall desirability & & \multicolumn{2}{|l|}{0.65} & \multicolumn{2}{|l|}{0.66} \\
\hline \multirow[t]{2}{*}{ Dependent variables } & Desirability & \multicolumn{2}{|c|}{ Optimized 1} & \multicolumn{2}{|c|}{ Optimized 2} \\
\hline & & Expected & Observed & Expected & Observed \\
\hline $\mathrm{Y}_{1}:$ LDPE crystallinity index & Minimize & 19.77 & 20.86 & 16.70 & 18.33 \\
\hline $\mathrm{Y}_{2}$ : LDPE carbonyl index & Maximize & 1.47 & 1.02 & 1.05 & 1.04 \\
\hline $\mathrm{Y}_{3}$ : LDPE weight loss (\%) & Maximize & 59.94 & 56.32 & 57.21 & 54.23 \\
\hline
\end{tabular}

Moreover, gel permeation chromatography (GPC) was performed to LDPE virgin and optimized REX treated residues before undergoing bio-adhesion testing. Analysis was carried out using Tosoh EcoSEC HT-GPC 220 (UK) using an established protocol for assaying. GPC analysis results are presented in Table 5 as values of the weight-average molecular weight (Mw), the number-average molecular weight (Mn), and polydispersity index (PDI). As shown in Table 5, a significant reduction in the Mw and Mn masses of LDPE valued by 5 folds for Mw and 3 folds in the case of Mn upon treatment using the proposed technique. Noticeably, no ultrahigh-Mw polymer was detected in the residual solid indicating the occurrence of polymer degradation reactions. The PDIs of optimized treated residues were also lower in comparison with virgin LDPE. 
Table 5

GPC analysis of pretreated LDPE

\begin{tabular}{|llll|}
\hline Sample & $\mathbf{M w}\left(\mathbf{1 0}^{\mathbf{3}} \mathbf{g} / \mathrm{mol}\right)$ & $\mathrm{Mn}\left(10^{3} \mathbf{g} / \mathrm{mol}\right)$ & PDI \\
\hline Virgin LDPE & 128.4 & 18.1 & 7.09 \\
\hline Optimized 1 & 31.1 & 6.4 & 4.85 \\
\hline Optimized 2 & 23.9 & 5.8 & 4.54 \\
\hline
\end{tabular}

\subsection{Bacterial adhesion and biofilm formation on optimized pretreated LDPE}

Pseudomonas species were previously shown to induce biodegradation of a variety of in treated plastics(s) [22]. Recently a consortium with Bacillus spp. was demonstrated to perform favourable biodegradation of the polyester polymer polyethylene terephthalate [6]. Here, P. aeruginosa was selected along with two environmental isolates of Bacillus sp. as opportunistic pathogens with substantial abilities to form biofilms [23]. In the current study, biofilms were successfully formed on pretreated LDPE samples; Optimized 1 and Optimized 2, after 14 days of incubation. Notably, adhesion and biofilm formation were 8- and 4-fold lower on untreated LDPE control samples (Figure 4[a]). Weight loss or change in the carbonyl and crystallinity indices of the LDPE samples during the 14 days of incubation biofilm attachment was not detected, indicating that while effective bio-adherence and bio-amenability has been demonstrated, evidence of biodegradation has not been detected. Interestingly, the consortium exhibited between 2-3-fold lower ability to form biofilms on all samples in comparison to the pure $P$. aerugionosa culture. This is in contrast to the similar study of Roberts et al, that used an environmental consortium of similar composition, and maybe associated with antagonistic effects amongst species [24]. The fact that Optimized 1 material showed a remarkable ability to adsorb basic, positively charged crystal violate (CV) stain [25], regardless of the presence of the microorganisms (Figure 4[b]), is evidence that the REX treatment induced beneficial surface changes which strongly diminish the bio-inert characteristics of LDPE, supporting a route towards effective sustainable post-consumer LDPE biodegradation.

\section{Conclusion}

Bioinert LDPE has been efficiently converted to bio-amenable LDPE using an energy favourable, and industrially scalable, fast high throughput REX assisted oxidation process. In comparison to virgin LDPE, ready biofilm formation was demonstrated for REX pretreated LDPE. The developed pretreatment process was operated for a duration of 5 minutes and optimized using BBD to provide independent variable values: low screw speed (50 rpm), operating temperature of 380-390 $₫ \mathrm{C}$ and the possibility of both non catalytic and catalytic conditions using $\mathrm{SiO}_{2}$ concentrations of ( 0 and $\left.2 \%(w / w)\right)$. FTIR and DSC spectra of the residual LDPE obtained after pretreatment with the REX assisted oxidation technique showed a significant increase in residual LDPE carbonyl index from 0 to 1.04 and a decrease of LDPE crystallinity 
index from 29 to 18. Additionally, GPC analysis revealed a significant decrease in the Mw of up to $81 \%$ for treated versus virgin LDPE confirming the occurrence of polymer chains breakdown during the REX process. Biofilms were successfully formed on pretreated LDPE samples after 14 days of incubation indicating their potential for further biodegradation. Conclusively, the proposed LDPE pretreatment approach using REX assisted oxidation technique followed by enhanced biofilm formation presents a high potential route towards the achievement of industrially scalable post-consumer LDPE biodegradation.

\section{Declarations}

\section{Availability of data and materials}

All data generated or analysed during this study are included in the article.

\section{Competing interests}

The authors declare no conflict of interest.

\section{Funding}

This project received funding from the European Union's Horizon 2020 research and innovation programme under grant agreement No. 870292 (BIOICEP)

\section{Authors' contributions}

P.F. and O.A. contributed equally in Conceptualization, Data curation, Investigation, Writing and reviewing original draft; M.A.V. and I.A practical experimenting; M.A. Analysis, Data curation; J.N.R. Validation, Writing original draft, M.B.F. Supervision, Validation, Writing original draft.

\section{Acknowledgements}

This project was supported by the National Natural Science Foundation of China (grant numbers: Institute of Microbiology, Chinese Academy of Sciences: 31961133016; Beijing Institute of Technology: 31961133015; Shandong University: 31961133014).

\section{References}

1. P. Europe, Plastics-The Facts 2020, PlasticEurope. 1 (2020) 1-64.

2. P.K. Roy, S. Titus, P. Surekha, E. Tulsi, C. Deshmukh, C. Rajagopal, Degradation of abiotically aged LDPE films containing pro-oxidant by bacterial consortium, Polym. Degrad. Stab. 93 (2008) 19171922.

3. E.R. Secchi, S. Zarzur, Plastic debris ingested by a Blainville's beaked whale, Mesoplodon densirostris, washed ashore in Brazil, Aquatic Mammals 25(1) (1999) 21-24 . 
4. L.B. Spear, D.G. Ainley, C.A. Ribic, Incidence of plastic in seabirds from the tropical pacific, 19841991: relation with distribution of species, sex, age, season, year and body weight, Mar. Environ. Res. 40 (1995) 123-146.

5. A.C. Palmisano, C.A. Pettigrew, Biodegradability of plastics, Bioscience. 42 (1992) 680-685.

6. C. Roberts, S. Edwards, M. Vague, R. León-Zayas, H. Scheffer, G. Chan, N.A. Swartz, J.L. Mellies, Environmental Consortium Containing Pseudomonas and Bacillus Species Synergistically Degrades Polyethylene Terephthalate Plastic, MSphere. 5 (2020). https://doi.org/10.1128/msphere.01151-20.

7. S.K. Sen, S. Raut, Microbial degradation of low density polyethylene (LDPE): A review, J. Environ. Chem. Eng. 3 (2015) 462-473.

8. S. Awasthi, N. Srivastava, T. Singh, D. Tiwary, P.K. Mishra, Biodegradation of thermally treated low density polyethylene by fungus Rhizopus oryzae NS 5, 3 Biotech. 7 (2017) 73.

9. G. Mathur, A. Mathur, R. Prasad, Colonization and degradation of thermally oxidized high-density polyethylene by Aspergillus niger (ITCC No. 6052) isolated from plastic waste dumpsite, Bioremediat. J. 15 (2011) 69-76.

10. A.C. Albertsson, Biodegradation of synthetic polymers. II. A limited microbial conversion of $14 \mathrm{C}$ [carbon isotope] in polyethylene to $14 \mathrm{CO}_{2}$ [carbon dioxide isotope] by some soil fungi, J. Appl. Polym. Sci. 22 (1978) 3419-3433.

11. J.H. Cornell, A.M. Kaplan, M.R. Rogers, Biodegradability of photooxidized polyalkylenes, J. Appl. Polym. Sci. 29 (1984) 2581-2597.

12. E. Chiellini, A. Corti, G. Swift, Biodegradation of thermally-oxidized, fragmented low-density polyethylenes, Polym. Degrad. Stab. 81 (2003) 341-351.

13. J. Aguado, D.P. Serrano, J.M. Escola, E. Garagorri, Catalytic conversion of low-density polyethylene using a continuous screw kiln reactor, Catal. Today. 75 (2002) 257-262.

14. S.A. Nield, C. Tzoganakis, H.M. Budman, Chemical modification of low density polyethylene through reactive extrusion: Part I: Process development and product characterization, Adv. Polym. Technol. J. Polym. Process. Inst. 19 (2000) 237-248.

15. M.D. Wallis, S.K. Bhatia, Thermal degradation of high density polyethylene in a reactive extruder, Polym. Degrad. Stab. 92 (2007) 1721-1729.

16. J. Shah, M.R. Jan, F. Mabood, F. Jabeen, Catalytic pyrolysis of LDPE leads to valuable resource recovery and reduction of waste problems, Energy Convers. Manag. 51 (2010) 2791-2801.

17. J.H. Merritt, D.E. Kadouri, G.A. O'Toole, Growing and analyzing static biofilms, Curr. Protoc. Microbiol. $22(2011) 1 B-1$.

18. S.S. Ali, I.A. Qazi, M. Arshad, Z. Khan, T.C. Voice, C.T. Mehmood, Photocatalytic degradation of low density polyethylene (LDPE) films using titania nanotubes, Environ. Nanotechnology, Monit. Manag. 5 (2016) 44-53. https://doi.org/10.1016/j.enmm.2016.01.001.

19. W. Li, C. Zhang, H. Chi, L. Li, T. Lan, P. Han, H. Chen, Y. Qin, Development of antimicrobial packaging film made from poly(lactic acid) incorporating titanium dioxide and silver nanoparticles, Molecules. 
22 (2017). https://doi.org/10.3390/molecules22071170.

20. T. Corrales, F. Catalina, C. Peinado, N.S. Allen, E. Fontan, Photooxidative and thermal degradation of polyethylenes: Interrelationship by chemiluminescence, thermal gravimetric analysis and FTIR data, J. Photochem. Photobiol. A Chem. 147 (2002) 213-224. https://doi.org/10.1016/S10106030(01)00629-3.

21. M. Ponjavic, M.S. Nikolic, J. Nikodinovic-Runic, S. Jeremic, S. Stevanovic, J. Djonlagic, Degradation behaviour of PCL/PEO/PCL and PCL/PEO block copolymers under controlled hydrolytic, enzymatic and composting conditions, Polym. Test. 57 (2017) 67-77. https://doi.org/10.1016/j.polymertesting.2016.11.018.

22. R.A. Wilkes, L. Aristilde, Degradation and metabolism of synthetic plastics and associated products by Pseudomonas sp.: capabilities and challenges, J. Appl. Microbiol. 123 (2017) 582-593. https://doi.org/10.1111/jam.13472.

23. M.T.T. Thi, D. Wibowo, B.H.A. Rehm, Pseudomonas aeruginosa biofilms, Int. J. Mol. Sci. 21 (2020) 125. https://doi.org/10.3390/ijms21228671.

24. M. Simões, L.C. Simoes, M.O. Pereira, M.J. Vieira, Antagonism between Bacillus cereus and Pseudomonas fluorescens in planktonic systems and in biofilms., Biofouling. 24 (2008) 339-349. https://doi.org/10.1080/08927010802239154.

25. A. Wanger, V. Chavez, R.S.P. Huang, A. Wahed, J.K. Actor, A. Dasgupta, Chapter 5 - Biochemical Tests and Staining Techniques for Microbial Identification, in: A. Wanger, V. Chavez, R.S.P. Huang, A. Wahed, J.K. Actor, A.B.T.-M. and M.D. in P. Dasgupta (Eds.), Elsevier, 2017: pp. 61-73. https://doi.org/https://doi.org/10.1016/B978-0-12-805351-5.00005-3.

\section{Figures}


Crystallinity index
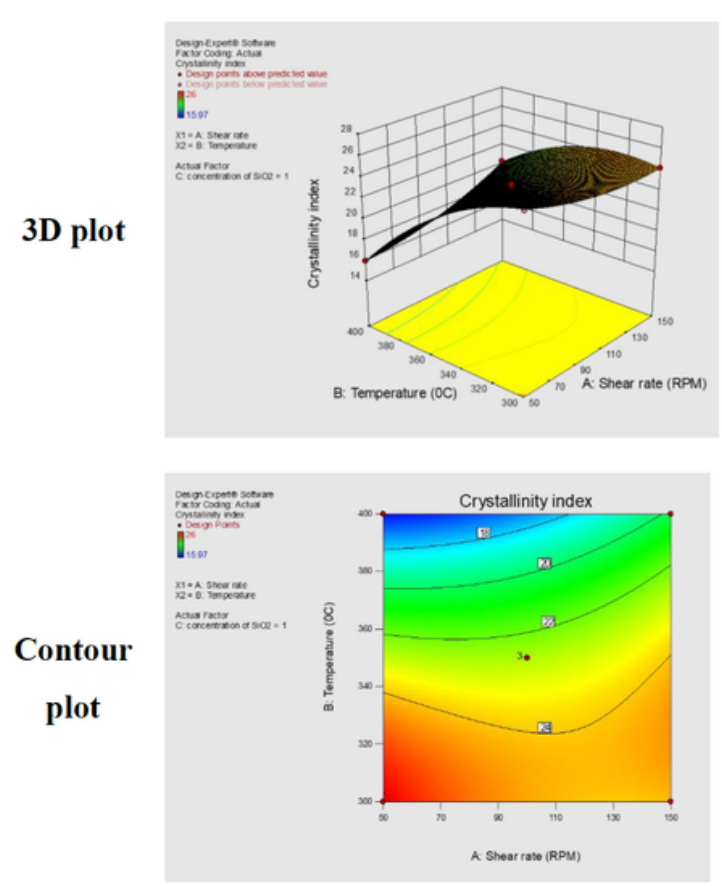

Carbonyl index
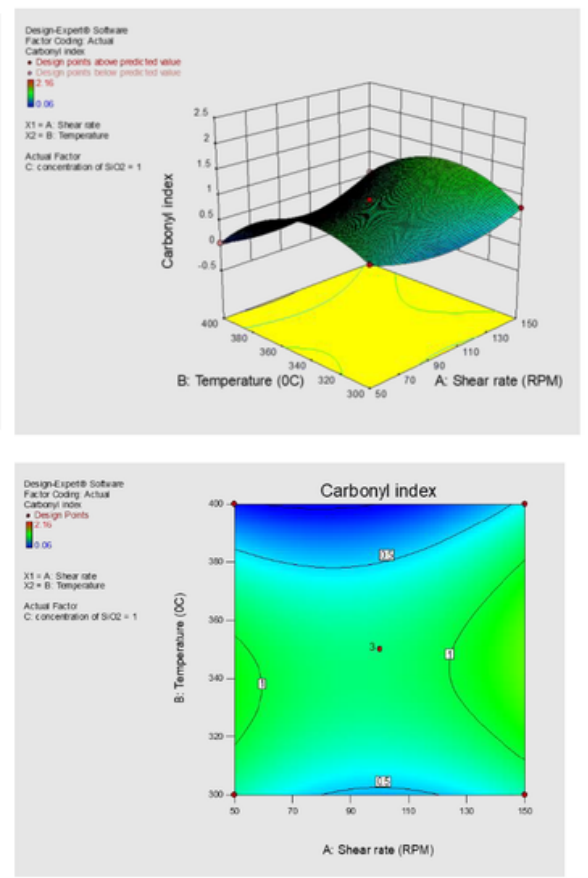

LDPE Weight loss (\%)
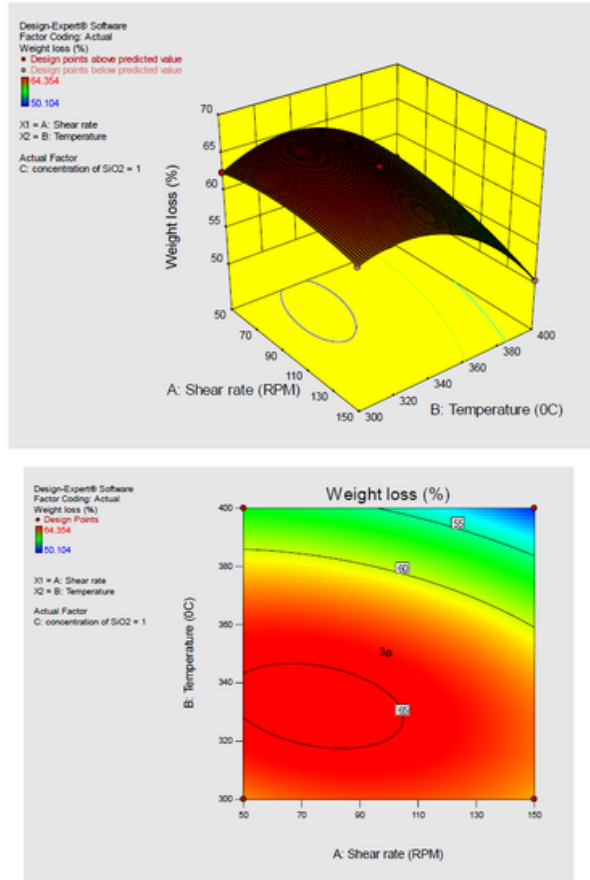

\section{Figure 1}

$3 \mathrm{D}$ and contour plots of the effect of the interaction of screws speed (X1) and operating temperature (X2) on crystallinity index, carbonyl index and LDPE weight loss (\%)

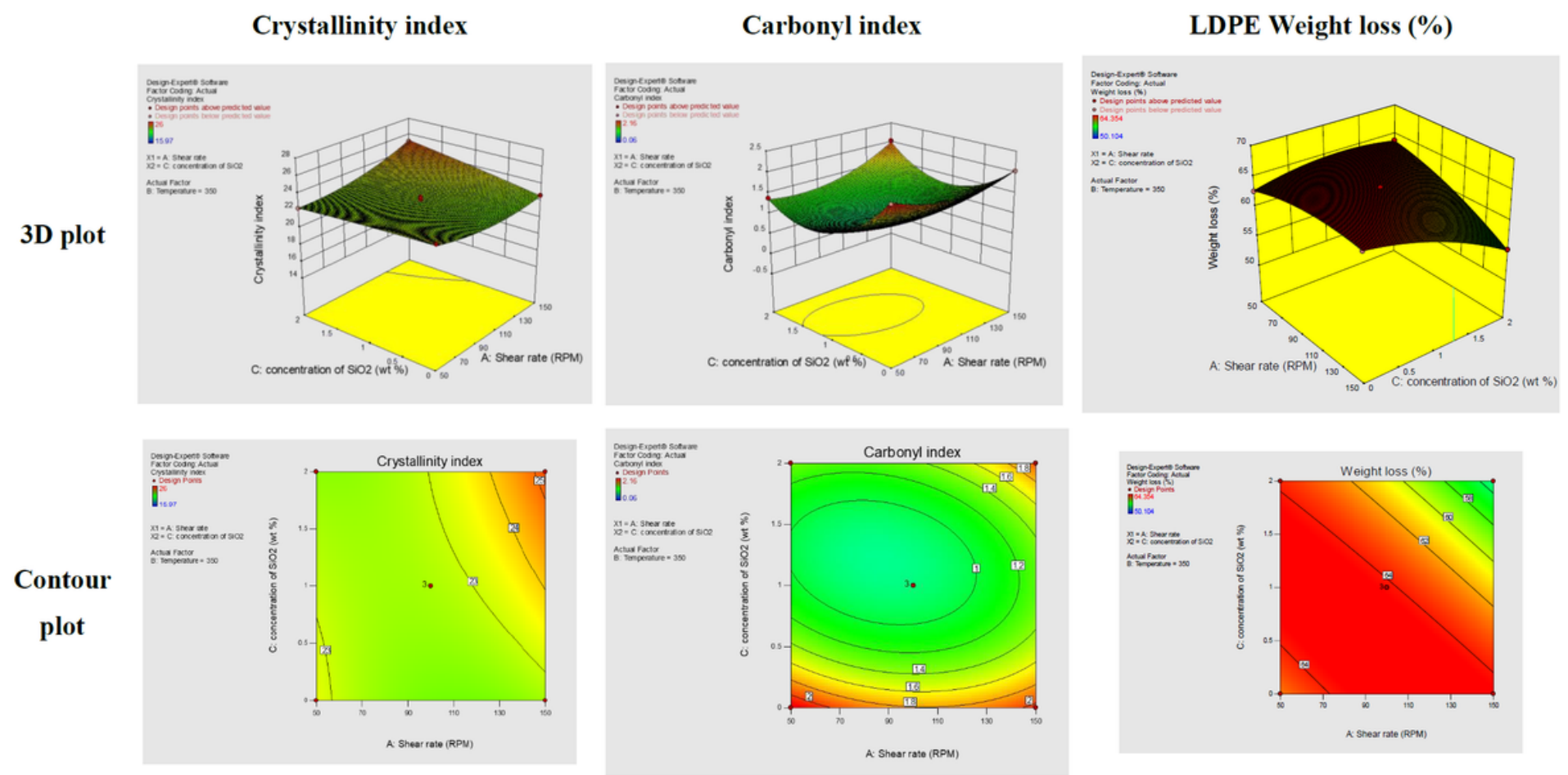

Figure 2 
3D and contour plots of the effect of the interaction of screws speed (X1) and concentration of SiO2 (X3) on crystallinity index, carbonyl index and LDPE weight loss (\%)

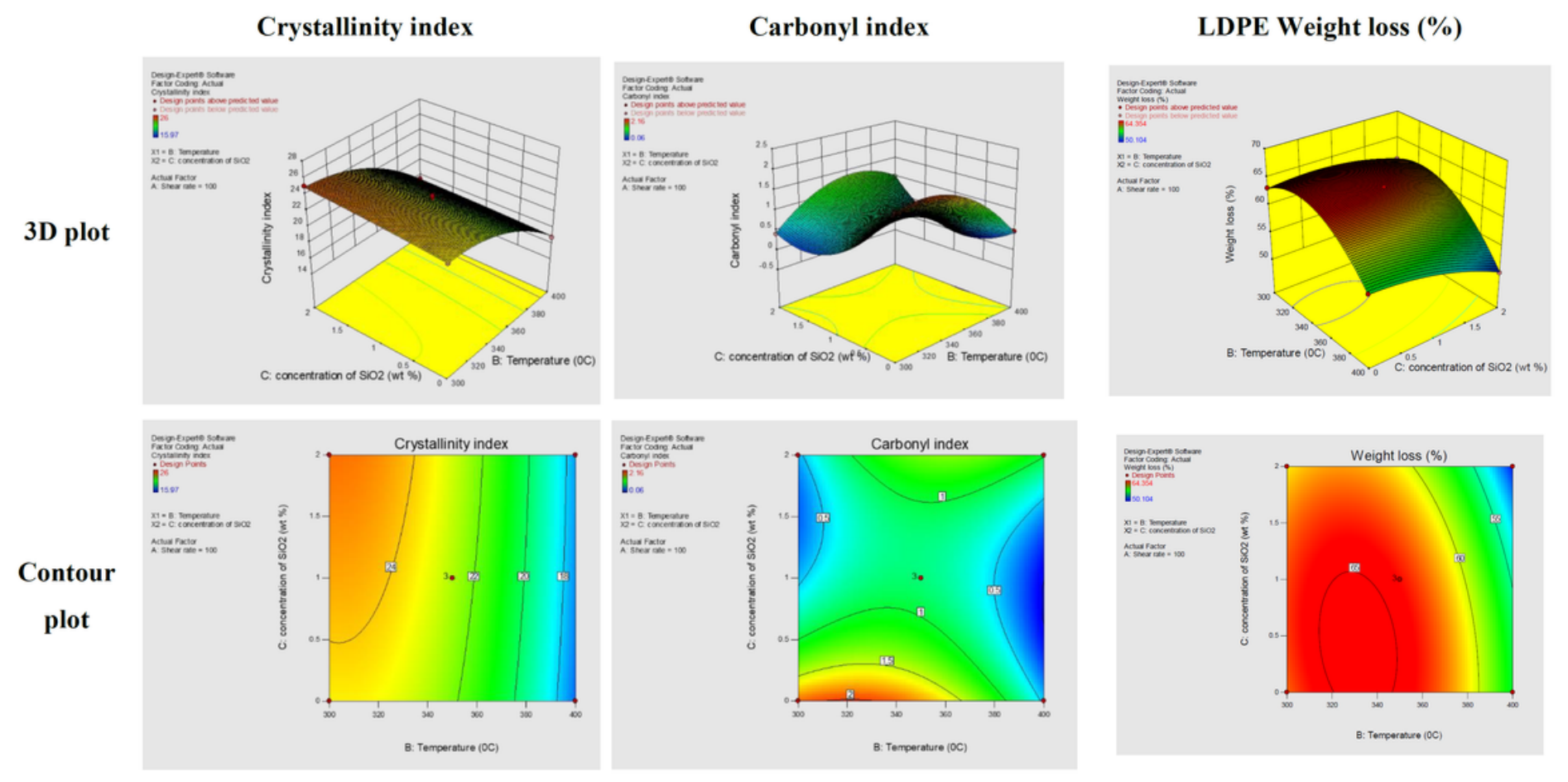

\section{Figure 3}

$3 \mathrm{D}$ and contour plots of the effect of the interaction of operating temperature (X2) and concentration of SiO2 (X3) on crystallinity index, carbonyl index and LDPE weight loss (\%) 
A)

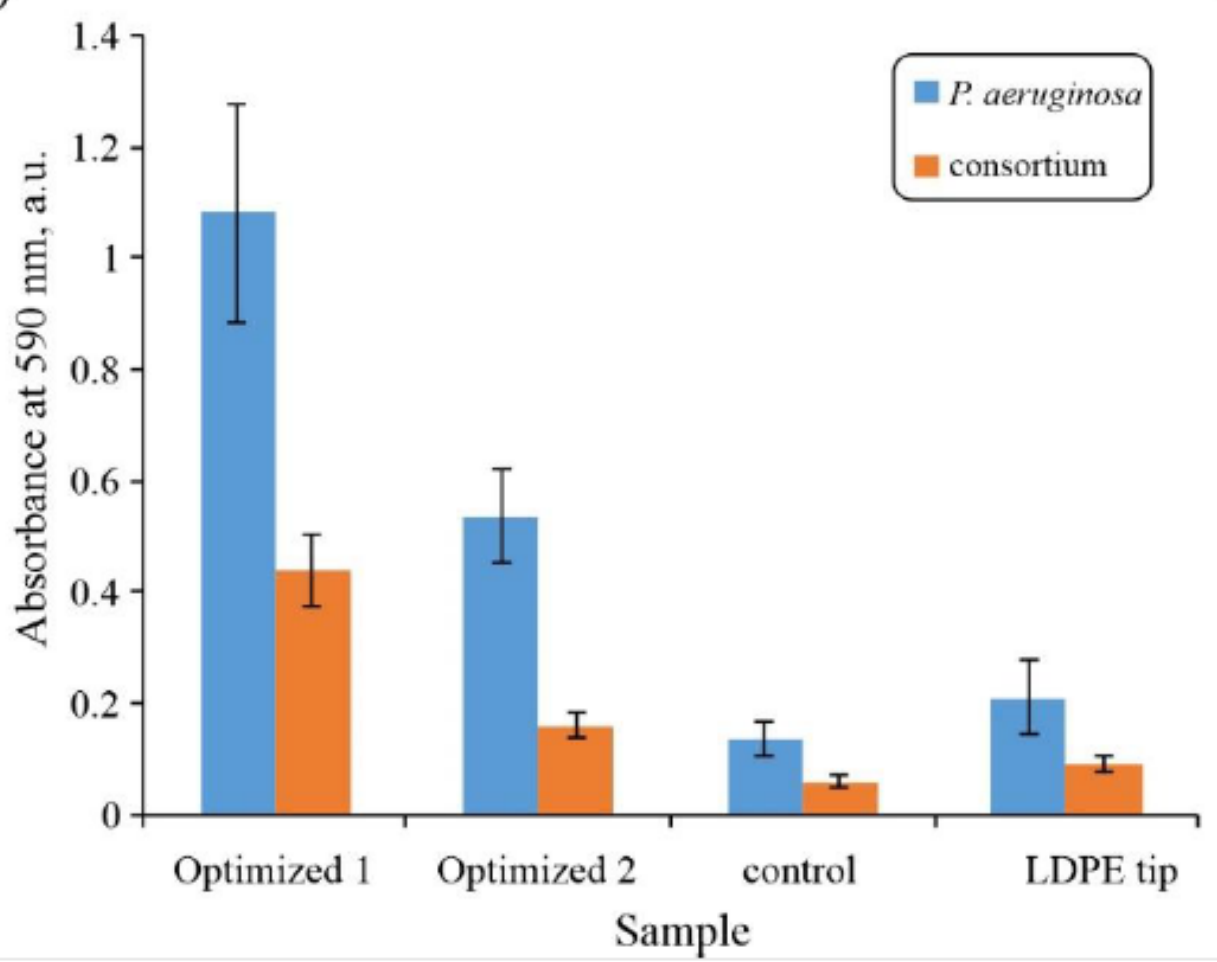

B)

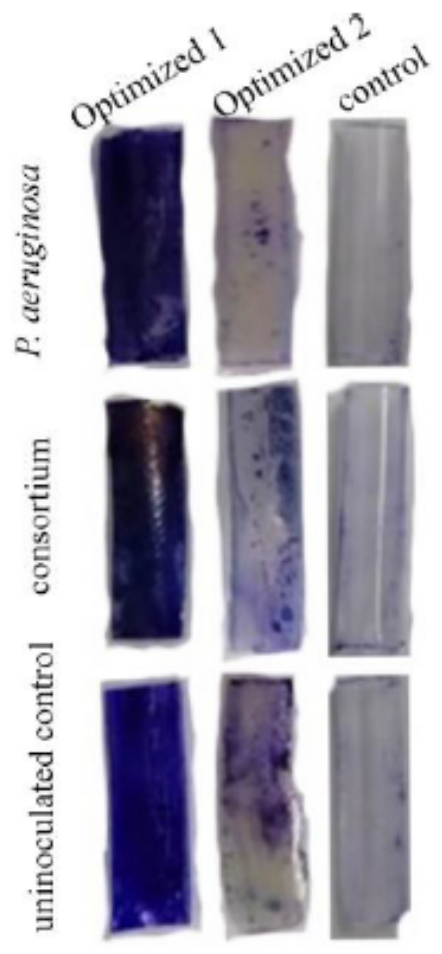

Figure 4

Biofilm formation on LDPE samples using P. aeruginosa pure culture and consortium consisting of $P$. aeruginosa and two Bacillus sp. after 14 days of incubation determined [a] spectrophotometrically using crystal violet straining; and [b] the appearance of the samples prior to distaining process. 